\title{
GLOBAL STABILITY FAILURE OF A 3D COMPOSITE STRUCTURE ACCOMPANIED BY UNAVOIDABLE POLYMORPHIC UNCERTAINTIES
}

\author{
Martin Drieschner ${ }^{1}$, Christoph Wolf ${ }^{1}$ and Yuri Petryna ${ }^{1}$ \\ ${ }^{1}$ Department of Civil Engineering, Technische Universität Berlin \\ Gustav-Meyer-Allee 25, 13355 Berlin, Germany \\ martin.drieschner@tu-berlin.de, c.wolf@tu-berlin.de, yuriy.petryna@tu-berlin.de, \\ www.statik.tu-berlin.de
}

Key words: Aleatory Uncertainty, Epistemic Uncertainty, Fuzzy-Stochastic Finite Element Method, Global Stability Failure, Carbon Fiber Reinforced Plastic (CFRP)

\begin{abstract}
In this study, a non-linear stability analysis of a carbon fiber reinforced plastic (CFRP) considering unavoidable polymorphic uncertainties is conducted. For the realistic incorporation of the uncertainties in the finite element model, thickness variations and geometrical inaccuracies have been detected in advance by non-destructive testing. For that, a structure made of CFRP has been designed. Additionally, the material parameters have been defined as dependent stochastic variables based on reference studies in the literature. The distinction in aleatory and epistemic uncertainties leads to different uncertainty models and to a computationally costly fuzzy-stochastic analysis. Strains and displacements have been measured in a symmetric three-point bending test and compared to the numerical predictions. In addition to the present uncertain parameters, a case study shows that the fiber volume content and a small pre-deformation should be taken into account to minimize the deviation from the experimental results.
\end{abstract}

\section{INTRODUCTION}

This study has been performed within the research project MuScaBlaDes: "Multi-scale failure analysis with polymorphic uncertainties for optimal design of rotor blades", which is part of the DFG Priority Program (SPP 1886) "Polymorphic uncertainty modelling for the numerical design of structures" started in 2016. Rotor blades of wind turbines are thin-walled spatial composite structures, see Fig. 1. They typically consist of two aerodynamic shells and a bearing structure inside, either flanges and shear web(s) or a box girder. Various failure mechanisms can occur in operation which have to be investigated in the design process [1]. In this study, the focus is on the global stability failure of structural components made of carbon fiber reinforced plastic (CFRP). For that, experimental and numerical studies have been conducted on a representative thin-walled structure like in $[7,13]$. The structure is called the omega shell, which is supported using steel profiles and a plate made of glass fiber reinforced plastic (GFRP), see Section 2. It is assumed that the buckling is mainly affected by material imperfections and geometrical inaccuracies [5]. The influence of the latter one on the stability failure of thin-walled structures has been numerically evaluated in [2]. They are unavoidable due to partly hand-made manufacturing processes. Non-destructive testing (NDT) has been used for measuring the geometrical inaccuracies, see Section 3. A finite element model has been created in which the present uncertainties have been incorporated. Due 


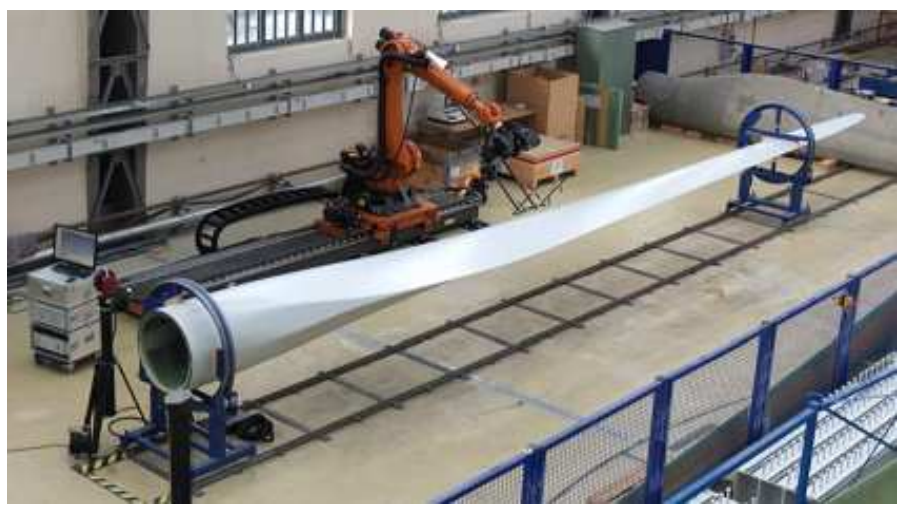

(a) Rotor blade at TU Berlin

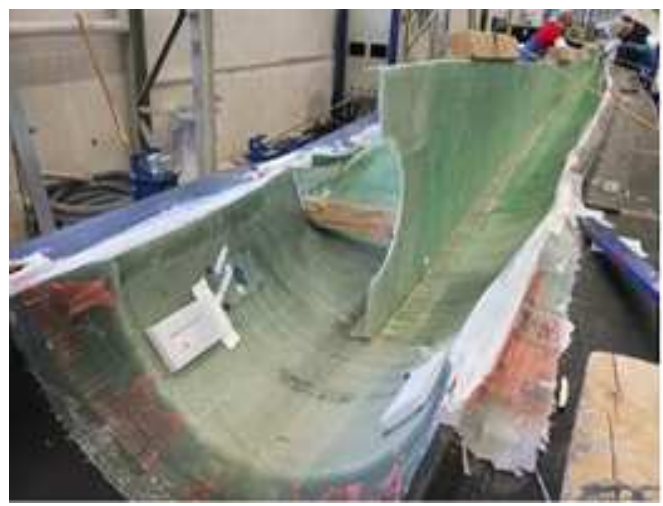

(b) Manufacturing process

Figure 1: Rotor blade of wind turbines as industrial example for thin-walled composite structures

to different uncertainty sources and knowledge about the uncertainties, a distinction in aleatory and epistemic uncertainties [8] with different uncertainty models is suggested as described in Section 4. The numerical results and their comparison with experimental results are presented in Section 5.

2 OMEGA SHELL

In order to observe the influence of numerous uncertainties on a numerically demanding problem a
structural component, a measurement procedure and an cxperimental routine has been clesigned and
implemented. Elastic buckling of a CFRP structure has been chosen, since it is strongly dependent
on various uncertainties, especially the geometrical imperfections (e.g. wall thickness). In order to
design an appropriate setup, fulfilling certain conditions is important, for example an easy and cost-
effective manufacturing incorporating CFRP materials, the possibility to observe buckling with optical measurement devices and creating a designated area for the buckles to form. The proposed structure, see

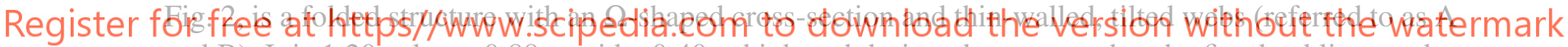
and B). It is $1.20 \mathrm{~m}$ long, $0.88 \mathrm{~m}$ wide, $0.40 \mathrm{~m}$ high and designed to ensure that the first buckling modes occur dominantly within the tilted webs. These are about half as thin as the remaining flanges (buckling strength is proportional to the third power of the wall thickness) and dominantly undergo compressive stress in a symmetric three-point-bending test. In a first iteration a quasi-isotropic laminate layering with a fiber volume content of $\varphi_{\text {des }}=55 \%$ has been chosen, consisting of a $\left[0^{\circ} / 45^{\circ} / 90^{\circ} /-45^{\circ}\right]_{\mathrm{S}}$ set-up for the webs and twice the amount for the flanges, yielding a web thickness of $t_{\mathrm{w}}=3.33 \mathrm{~mm}$ and a flange thickness of $t_{\mathrm{f}}=6.66 \mathrm{~mm}$. The calculated mass for the whole CFRP structure (without GFRP plate and edge reinforcements) is $m_{\mathrm{des}}=9.49 \mathrm{~kg}$. The mechanical properties of the carbon fibers (Tenax SSTS40 F13 F24K 1600tex from Teijin Carbon Europe GmbH [11]) and the matrix material (EPL 20 epoxy resin and EPH 161 hardener from R\&G Faserverbundwerkstoffe $\mathrm{GmbH}$ [9]) are presented in Table 1. The design has been made in cooperation with IPF Dresden. As a local partner, they also friendly undertook the manufacturing.

The finished composite shell structure is presented in Fig. 3a. To prevent premature buckling of the whole structure due to the free edges of the tilted webs, they are strengthened using specially designed edge supports made of steel U-profiles, see Fig. $3 \mathrm{~b}$ for reference. They can seamlessly slide onto the free 
Table 1: Mechanical properties of fibers and matrix material

\begin{tabular}{lrr}
\hline Mechanical property & Fiber & Matrix \\
\hline$E_{\|}$ & $238000 \mathrm{MPa}$ & $3150 \mathrm{MPa}$ \\
$E_{\perp}$ & $16000 \mathrm{MPa}$ & $3150 \mathrm{MPa}$ \\
$G_{\perp \|}$ & $50000 \mathrm{MPa}$ & $1150 \mathrm{MPa}$ \\
$v_{\perp \|}$ & 0.270 & 0.370 \\
$\varrho$ & $1.77 \mathrm{~g} \mathrm{~cm}^{-3}$ & $1.19 \mathrm{~g} \mathrm{~cm}^{-3}$ \\
$\alpha_{\|}^{\mathrm{T}}$ & $-4.55 \cdot 10^{-7} \mathrm{~K}^{-1}$ & $7.00 \cdot 10^{-5} \mathrm{~K}^{-1}$ \\
\hline
\end{tabular}
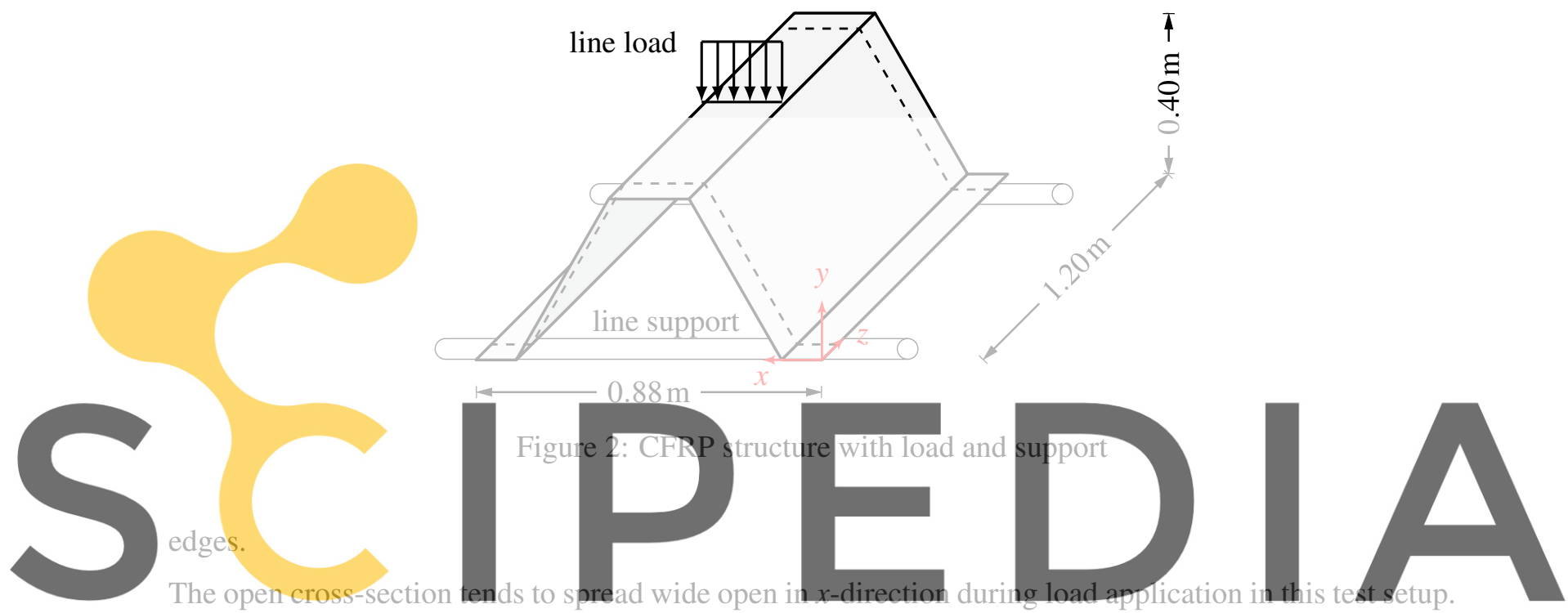

To counter this problem, a GFRP plate is glued to the bottom of the structure. Due to the high in-plane

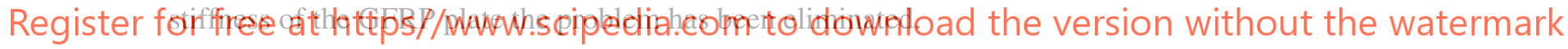

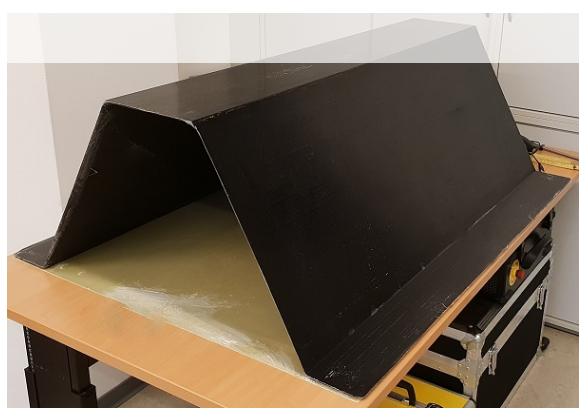

(a) Assembled CFRP structure with GFRP plate

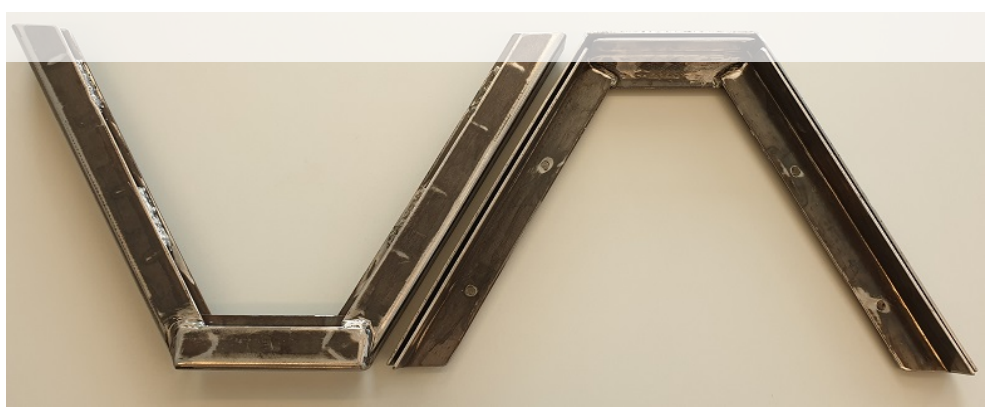

(b) Steel reinforcements to support both free edges against premature buckling

Figure 3: Omega shell and edge reinforcement 


\section{MEASUREMENTS AND EXPERIMENTAL SETUP}

The focus in the presented work is on the measurement of geometrical imperfections, namely dimensional deviations of the overall geometry and the wall thickness, since they have a significant influence on the buckling behavior [5]. Other imperfections, like material or physical imperfections (for example air voids within the matrix, initial cracks, variation of material parameters) have not been measured. Nonetheless, they also tend to have an impact on the buckling behavior, see Section 5.

After finishing the manufacturing process an initial inspection of the omega shell has been carried out. Particularly noteworthy is the rather smooth and even surface on the outside, while the inside one is more uneven and bumpy. The true weight of the structure without the GFRP plate is $m_{\text {true }}=10.78 \mathrm{~kg}$. Since the mass of fibers within the matrix is fixed one can recalculate the true fiber volume content to $\varphi_{\text {true }}=47 \%$.

\subsection{Ultrasonic thickness measurement}

As previously mentioned, the inside surface of the omega shell is not smooth and rather uneven up to a certain degree due to the manufacturing process. Since the web thickness is only a few millimeters, even small variations can have a meaningful influence on the buckling behavior. The actual thickness measurements have been performed using the ultrasonic pulse-echo system MUSE Z-400. Both web areas have been scanned with a minimal resolution of $150 \mu \mathrm{m}$ resulting in over 44 million data points per square meter. The overall results are displayed in Fig. 4, where the thickness of the webs is amplified by
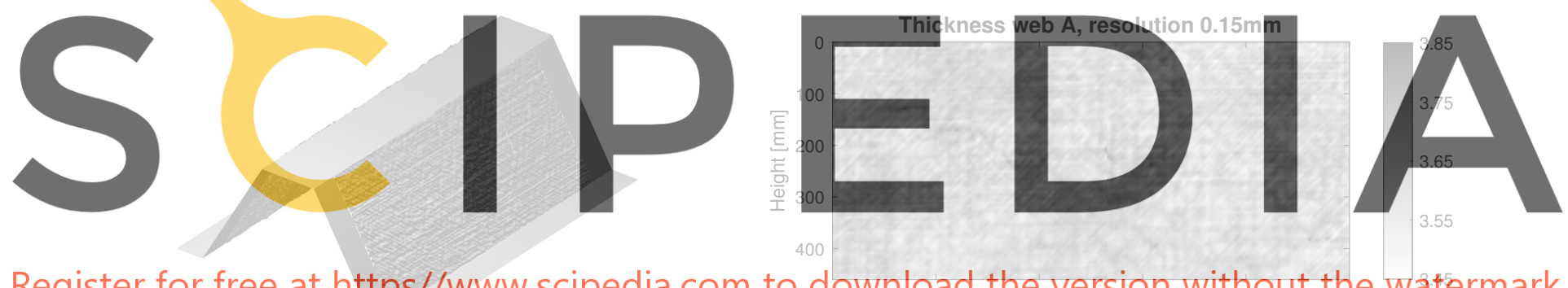

Register for free at https//www.scipedia.com to download the eversign witbout the watermark

(a) Measured web thickness on web A

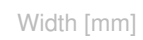

and $\mathrm{B}$ amplified by a factor of 50

(b) Thickness distribution of web $\mathrm{A}$ in millimeters

Figure 4: Ultrasonic measurement of the web thickness

a factor of 50. Due to some mounting limitations of the measurement device, it has not been possible to cover the remaining $10 \mathrm{~cm}$ in front of every web edge. The mean thickness of both webs $\mu_{\text {true }} \approx 3.61 \mathrm{~mm}$ is about $8 \%$ larger than the designed thickness, see Fig. 8.

\subsection{Global geometry measurement using fringe projection method}

An optical measurement using the fringe projection method has been used to get information about the actual shape of the structure. Here, a specific pattern of different fringes is projected onto an object and measured using a calibrated camera. After processing the results are presented as triangular mesh of the geometry. This data can be analyzed further or compared to the designed geometry. The deviation of the 
real geometry from the design is presented in Fig. 5.

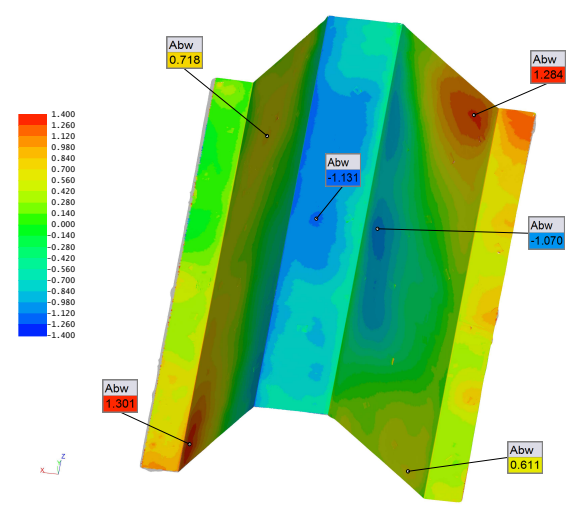

Figure 5: Deviations from design geometry in millimeters

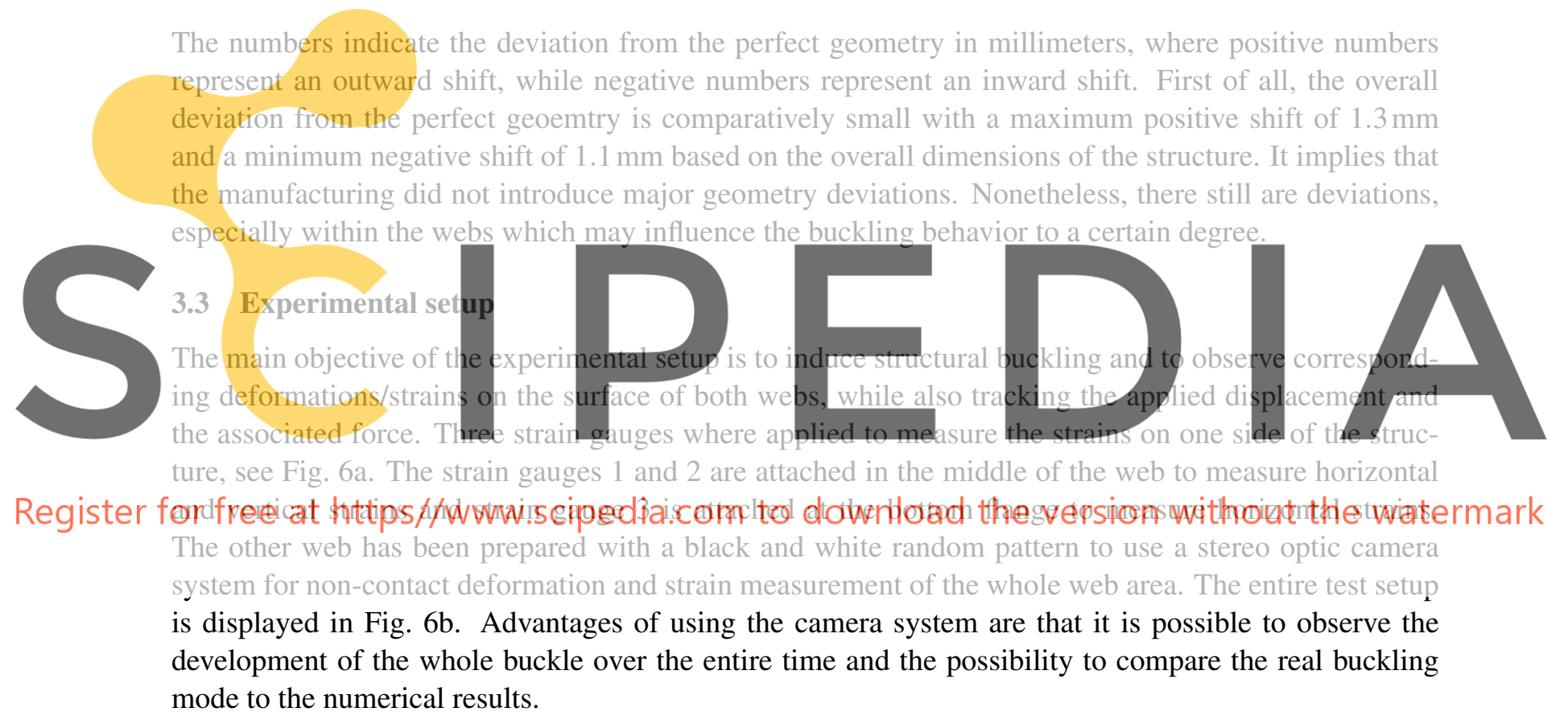

The entire experiment is conducted displacement-controlled with a constant rate of $500 \mu \mathrm{m} / \mathrm{min}$. This way it is possible to observe the post-buckling behavior of the structure, in case the structure is loosing stiffness on a certain path. The whole test has lasted about $30 \mathrm{~min}$ including some short interruptions. The structure violently failed at a vertical load of $45.36 \mathrm{kN}$ and a vertical displacement of $11.62 \mathrm{~mm}$ with an abrupt crack formation on the inside of the bottom of the upper flange. It was clearly visible during the test that the structure has buckled almost symmetrically with major deformations on both webs.

\section{UNCERTAINTY MODELS}

Unavoidable uncertainties are present and have to be considered in the numerical investigations. Some of them are irreducible due to randomness and natural variability. Statistical information are at disposal 


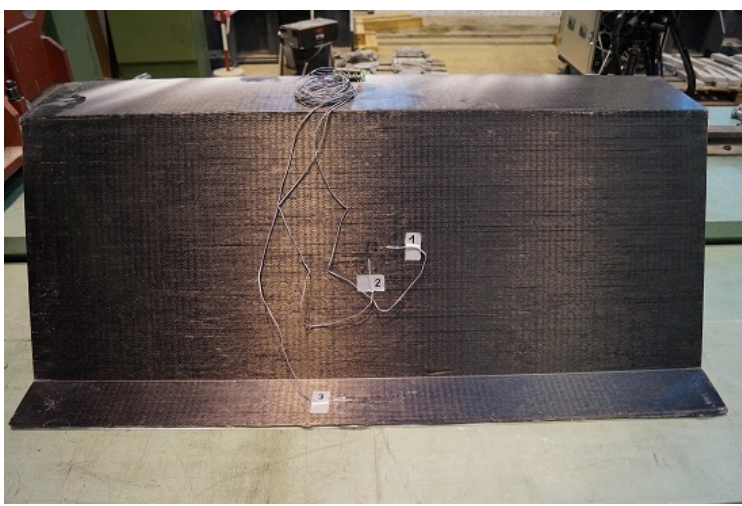

(a) Strain gauge arrangement (1: web B horizontal, 2: web B vertical, 3: flange horizontal)

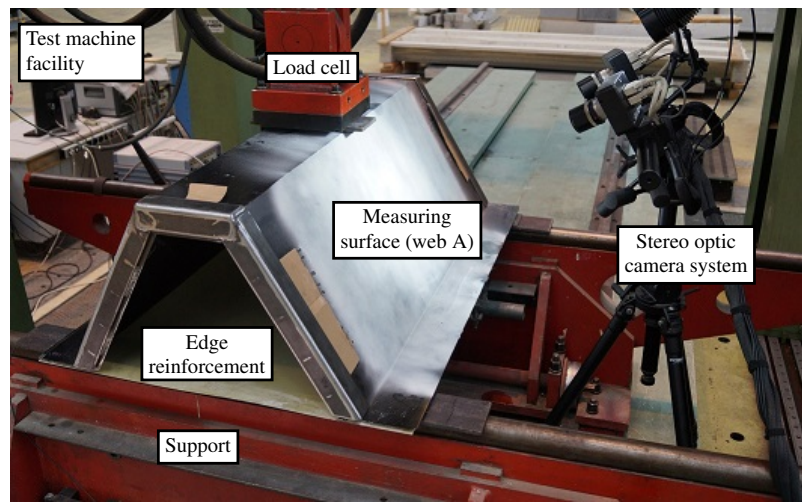

(b) Operational test setup with stereo optic camera system for deformation/strain measurement on web A

Figure 6: Strain gauge arrangement and test setup overview

in the literature or from conducted non-destructive testing. These uncertainties are called aleatory [8] and are quantified by random variables. In this study, lognormal distribution $\mathcal{L} \mathcal{N}(\mu, \sigma)$ with mean value of logarithmic values $\mu$ and standard deviation of logarithmic values $\sigma$ are used for all random variables, see Fig. 7a. Some uncertain parameters are based on limited amount of data, subjectivity or expert knowledge and no statistical information is given which could reduce the uncertainty. These uncertainties

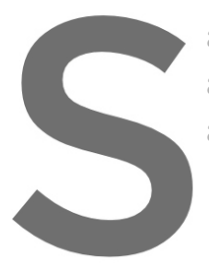
are called epistemic [8] an a triangular membership functior and core value $C=b$ are used, se
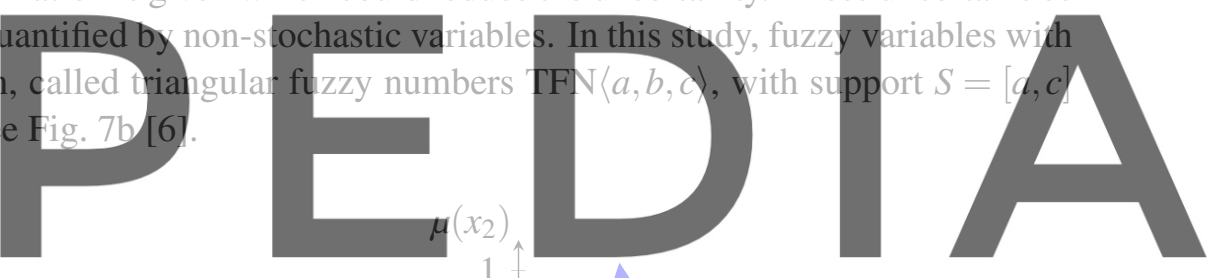

Register for
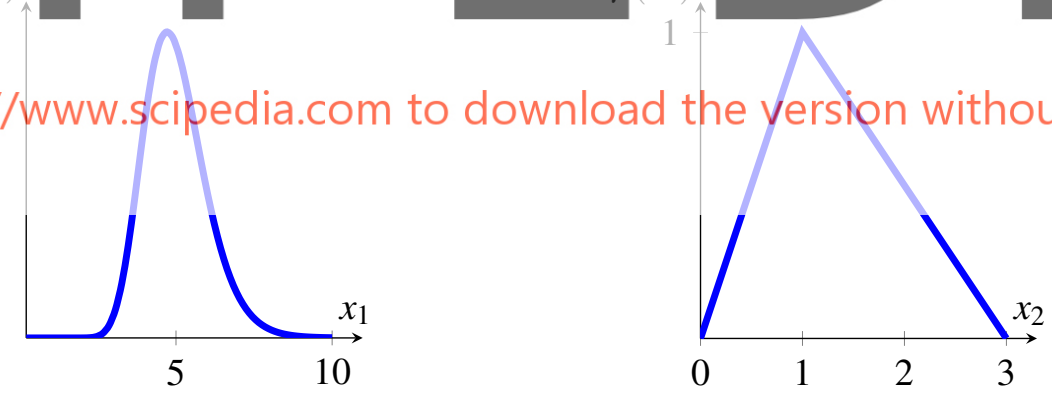

(a) Lognormal distribution:

(b) Triangular fuzzy number: $x_{1} \sim \mathcal{L} \mathcal{N}(\mu=5, \sigma=1)$ $x_{2}=\operatorname{TFN}\langle a=0, b=1, c=3\rangle$

Figure 7: Uncertainty models

\subsection{Material parameters}

The omega shell is made of carbon fiber reinforced plastic (CFRP) which is a multilayer composite consisting of individual unidirectional fiber layers. For each of them, transverse isotropy is assumed. The 
material parameters depends on the fiber (f) and matrix (m) properties which have been experimentally determined [14]. HTS carbon fibers (f) [11] and a matrix (m) [9] are used. The fiber and matrix properties are given in Table 1.

Based on [10], the material parameters $E_{\|}, E_{\perp}, v_{\perp \|}$ and $G_{\perp \|}$ and further dependent material parameters $v_{\| \perp}, v_{\perp \perp}$ and $G_{\perp \perp}$ of the unidirectional layer can be calculated, see Table 2. A fiber volume content of $\varphi_{\text {des }}=55 \%$ or $\varphi_{\text {true }}=47 \%$ has been used, respectively. The influence of a reduction of $E_{\perp}$ and $G_{\perp \|}$ on the results caused by material non-linear effects $[12,3]$ has been investigated. Only small deviations have been observed, so the material non-linearity is negligible and has been excluded in the following. In [15], statistical information of the mechanical properties of CFRP is given. By using the coefficients of variation (COV), lognormal distributions have been defined, see also Table 2.

Table 2: Material parameters of the carbon fiber reinforced plastic (CFRP)

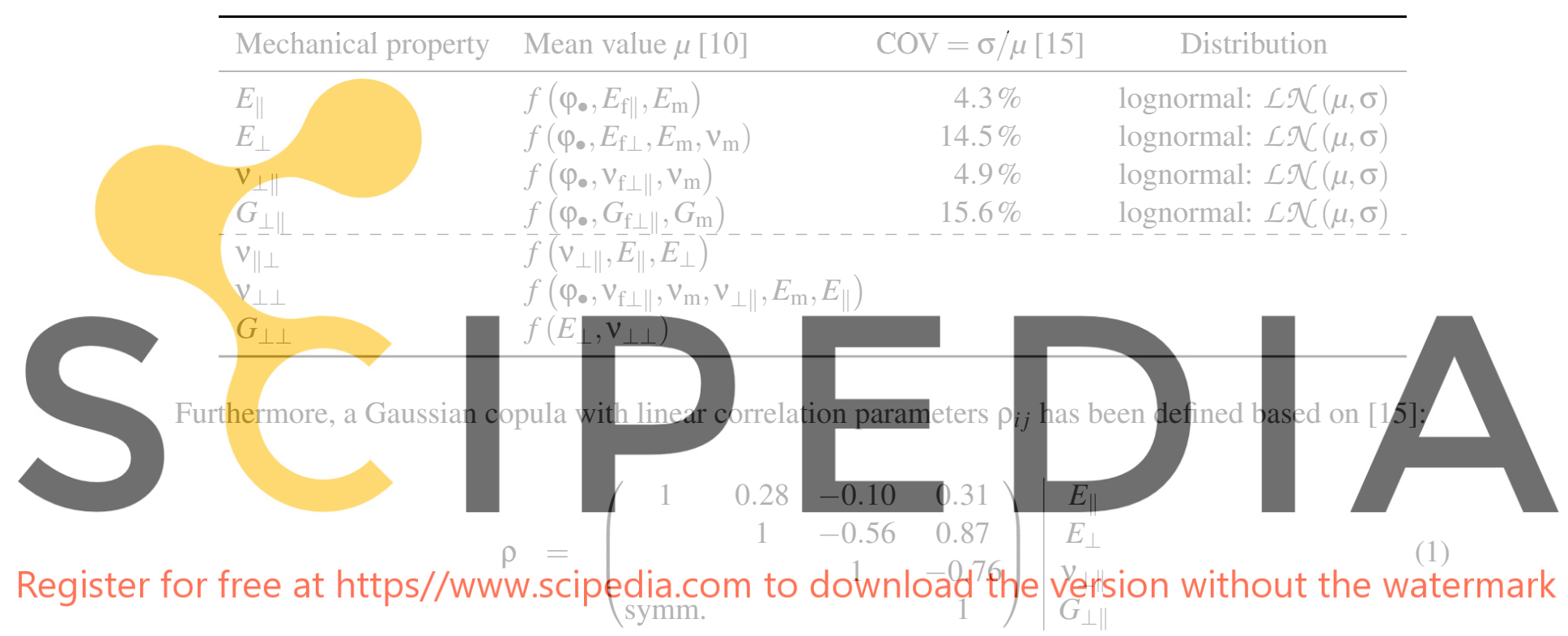

Inaccuracies in the manufacturing lead to imperfect undulated unidirectional layers. The undulation is considered by reducing the stiffness in longitudinal direction $E_{\|}$for which a factor $r_{\|} \approx 90 \%$ is usual [14]. In the following, the fuzzy variable $r_{\|}=\operatorname{TFN}\langle 80,90,95\rangle \%$ is defined.

\subsection{Web thickness}

The omega shell been has been designed with a thickness of $t_{\mathrm{des}}=3.33 \mathrm{~mm}$ for both webs (A and B). It could be measured by ultrasonic scanning, see Fig. 4, that the real thickness is spatially varying and about $8 \%$ larger. All measured thicknesses are displayed in a histogram, see Fig. 8 . The mean value for both webs is $\mu_{\text {true }} \approx 3.61 \mathrm{~mm}$ and the coefficient of variation is around $1 \%$. Based on that, a lognormal distribution for the web thickness $t_{\mathrm{w}} \sim \mathcal{L} \mathcal{N}(3.61,0.0361) \mathrm{mm}$ has been defined. It is worth mentioning that the increase of the mean value is more important for the stability analysis of the webs than the small spatial deviations. Thus, a spatially constant thickness $t_{\mathrm{w}}$ has been defined in this study. An extension to a (fuzzy-)random field definition is possible and considered in ongoing studies. 


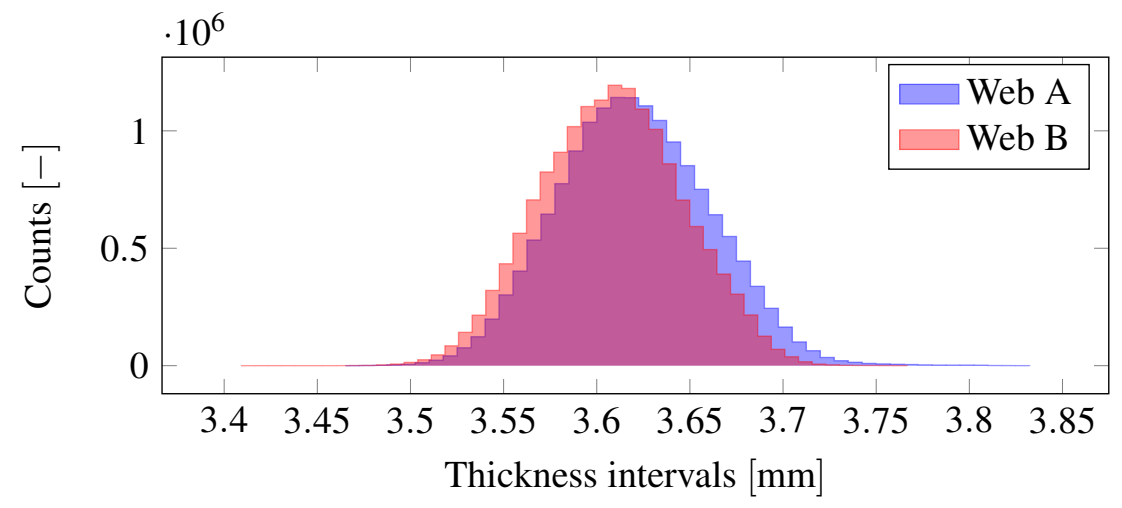

Figure 8: Web thickness: histogram of measurement data using a measurement resolution of $150 \mu \mathrm{m}$

\subsection{Global geometry deviation}

In addition to the web thickness, the global geometry has been measured and deviations from the original design could been determined, see Fig. 5. The deviations are considered in two different ways.

On the one hand, triangular fuzzy numbers are defined for the offsets of the web edges and the upper flange which are probably caused by "spring-in effects" in the manufacturing process [14]. Two opposite web edges have an offset of $\approx 1.3 \mathrm{~mm}$, the other ones of $\approx 0.7 \mathrm{~mm}$ and the upper flange of $\approx 1.1 \mathrm{~mm}$. To investigate the influence of the offsets, triangular fuzzy numbers $\Delta_{\mathrm{w}}=\operatorname{TFN}\langle 0,0,1.50\rangle \mathrm{mm}, \Delta_{\mathrm{wB}}=$ TFN $\langle 0,0,0.75\rangle \mathrm{mm}$ and $\left.\Delta_{\mathbf{u f f}}=\mathrm{THN} /-1.5,0,0\right\rangle \mathrm{mm}$ are defined with no offset on the cord and maximum offset on the support.

On the other hand, the deformed geometry accorcton $u_{\mathrm{imp}}$ can explicitly be given, so a case study has
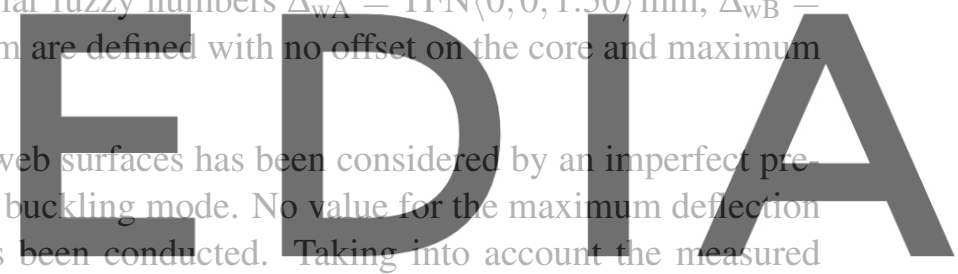
geometry in Fig. 5 with small deviations, a perfect geometry and a pre-deformed geometry with a value

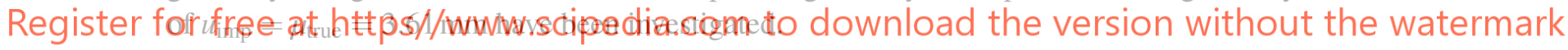

\subsection{Edge support area}

The omega shell has been reinforced on both longitudinal edges by steel U-profiles, see Section 2. For both webs (A and B) and the upper flange, the length of the support area is uncertain. It is assumed, that the reinforcement has an effect at least between $30 \%$ and $70 \%$ of the length. The maximum range has been specified between 5\% and 95\%. Each of the procentual start values $a_{\mathrm{wA}}, a_{\mathrm{wB}}$ and $a_{\mathrm{uf}}$ is quantified by $a_{\bullet}=\mathrm{TFN}\langle 5,30,30\rangle \%$ and each of the procentual end values $b_{\mathrm{wA}}, b_{\mathrm{wB}}$ and $b_{\mathrm{uf}}$ is quantified by $b_{\bullet}=$ TFN $\langle 70,70,95\rangle \%$.

\section{RESULTS}

Defining stochastic variables for aleatory uncertainties and fuzzy variables for epistemic uncertainties simultaneously leads to a nested fuzzy-stochastic analysis. The deterministic finite element model is embedded in the uncertainty space, which generally consists of the stochastic space, the interval space and the fuzzy space. In this approach, the interval space is empty and has not to be considered. It is usual 
that the stochastic space is embedded in the fuzzy space. The computational costs can be calculated as $t_{\mathrm{tot}}=n_{\mathrm{tot}} \cdot t_{\mathrm{D}}=n_{\mathrm{F}} \cdot n_{\mathrm{S}} \cdot t_{\mathrm{D}}$ with the number of samples in the fuzzy space $n_{\mathrm{F}}$, the number of samples in the stochastic space $n_{\mathrm{S}}$ and the duration $t_{\mathrm{D}}$ of the non-linear stability analysis of the deterministic model.

The deterministic model is created in the finite element program ANSYS and the complete numerical analysis is conducted in MATLAB, mainly by using the framework PolyUQ [4]. In the fuzzy space, the reduced transformation method [6] with five equidistantly distributed $\alpha$-levels and a priori fuzzy input dependencies is used. In the stochastic space, the classical Monte-Carlo method with $n_{\mathrm{S}}=200$ samples on each fuzzy sample is conducted.

In the following, the experimental and numerical results for the horizontal strain $\epsilon_{1, \mathrm{z}}$ at position 1 and for the displacement normal to the web surface $u_{\text {norm }}$ are presented. For the numerical investigations, three cases have been considered:

case $1 \varphi_{\text {des }}=55 \%$, perfect geometry

case $2 \varphi_{\text {true }}=47 \%$, perfect geometry

case $3 \varphi_{\text {true }}=47 \%$, pre-deformed geometry according to the symmetrical buckling mode with maximum deflection of $u_{\mathrm{imp}}=\mu_{\text {true }}=3.61 \mathrm{~mm}$ outward on both web surfaces A and $\mathrm{B}$

For all three cases, the mean value and the standard deviation have been calculated in the stochastic space. For both, the membership function $(\mu)$ can be shown and the center of gravity (COG) have been selected as defuzzified value in the fuzzy space.

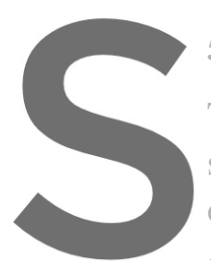

5.1 Strain gauge 1

The horizontal strain at posi

see Fig. 9a and 9b. The

on the support in case 3

$-45.36 \mathrm{kN}$ has been increased comp
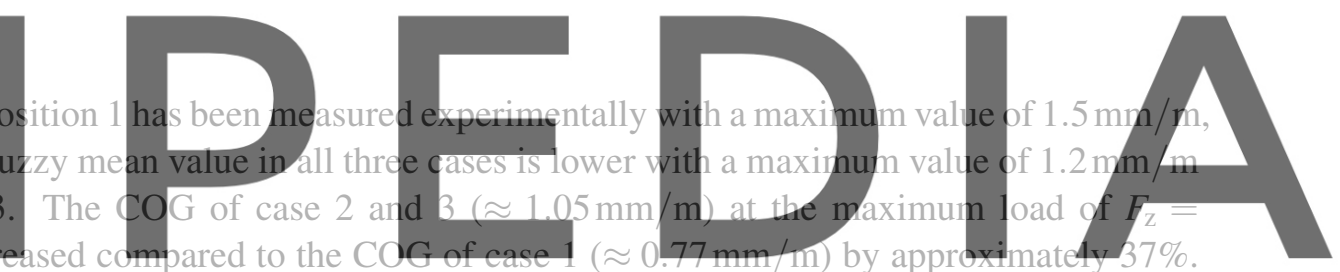

Furthermore, case 3 with the pre-deformed geometry is more appropriated to retrace the experimentally

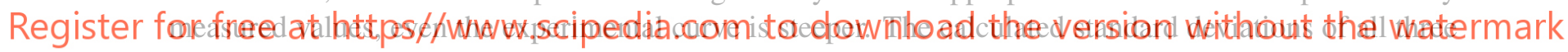
cases are comparable and are increasing by higher load values $F_{\mathrm{Z}}$, see Fig. 9c. The range at the maximum load is between $0.06 \mathrm{~mm} / \mathrm{m}$ and $0.15 \mathrm{~mm} / \mathrm{m}$, see Fig. $9 \mathrm{~d}$, which results in a coefficent of variation of approximately $10 \%$.

\subsection{Stereo optic camera system}

The displacement fields just before failure are depicted in Fig. 10a and 10b. They are qualitatively quite similar. The maximum deflection is in the upper part of the web and the buckle is stretched in horizonal direction. In the experiment, the structure has collapsed then with an abrupt crack formation on the inside of the bottom of the upper flange. The evaluation of the displacement in the center of the web $u_{\mathrm{M} \text {,norm }}$ is shown in Fig. 10c and 10d. Also here, the experimental curve is steeper than the numerical curves and the adjustments in case 2 and case 3 increases the displacement values, see Fig. 10c. The small support interval of approximately $2.5 \mathrm{~mm}$ in all three cases in Fig. 10d implies a small influence of the fuzzy input parameters on the result. 


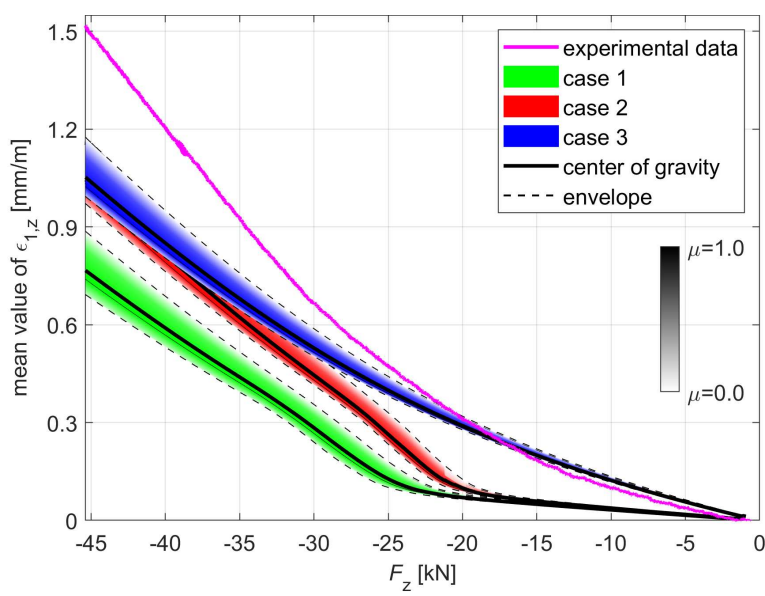

(a) fuzzy mean value of $\epsilon_{1, z}$

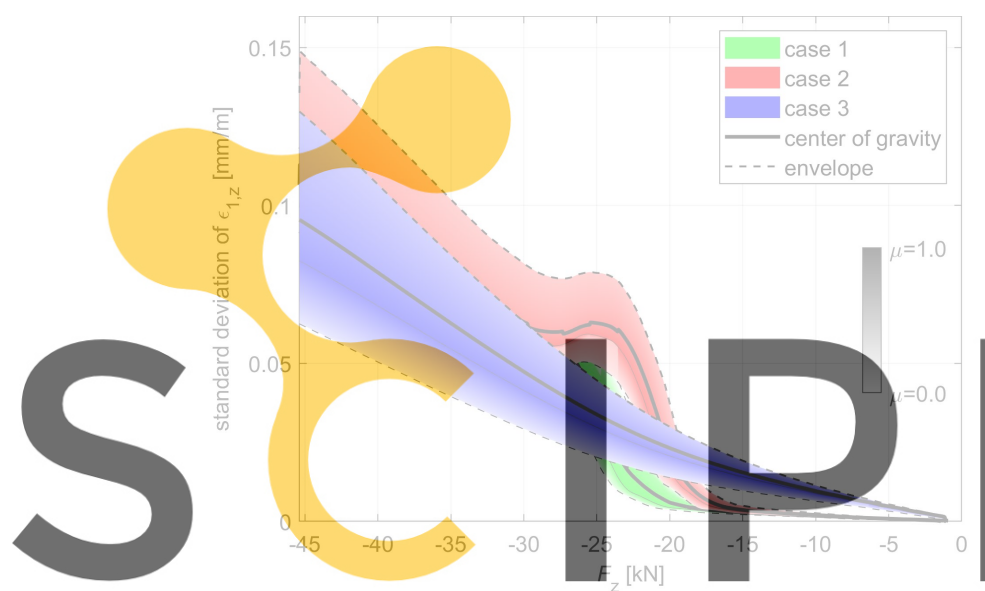

(c) fuzzy standard deviation of $\epsilon_{1, z}$

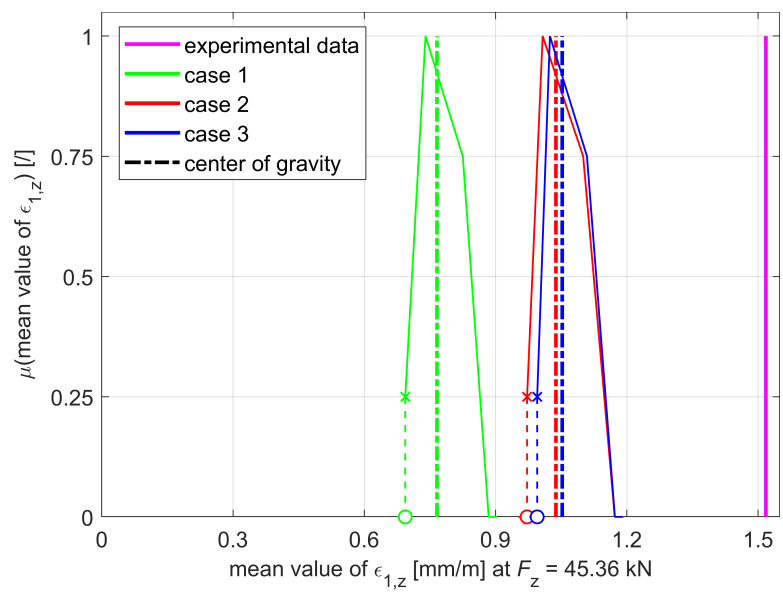

(b) membership function at $F_{\mathrm{z}}=-45.36 \mathrm{kN}$

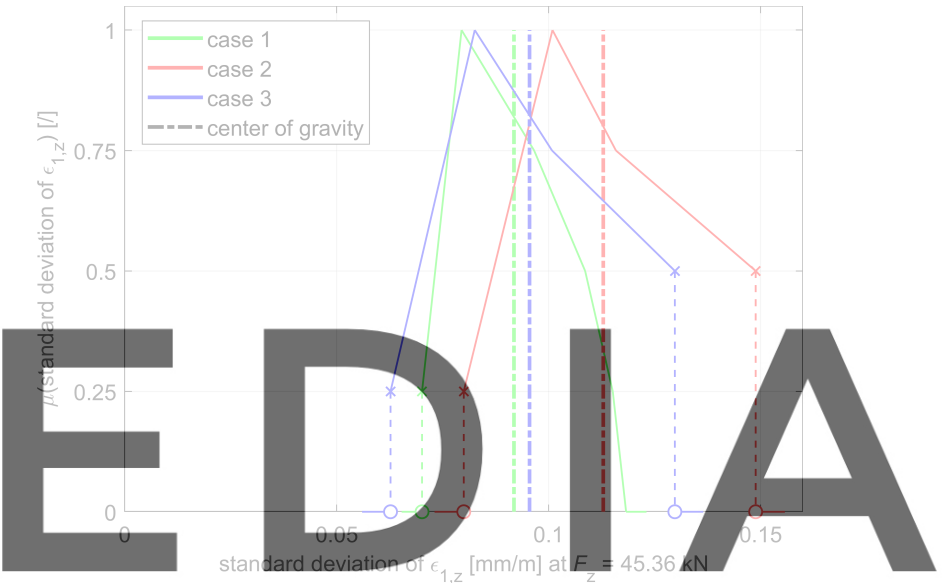

(d) membership function at $F_{\mathrm{z}}=-45.36 \mathrm{kN}$

Register for free at https//www.scipedia.com to download the version without the watermark

Figure 9: Fuzzy-stochastic output and experimental result for horizontal strain at position $1\left(\epsilon_{1, z}\right)$

\section{CONCLUSIONS}

In the present study, the global stability failure of a three-dimensional composite structure in the presence of unavoidable polymorphic uncertainties is investigated numerically as well as experimentally. The structure is a thin-walled carbon fiber reinforced shell with an omega cross-section. The shell has been manufactured, measured and loaded until failure in a symmetric three-point bending test. The measured uncertainties are incorporated in the numerical model by stochastic variables. Other uncertainties are only vaguely given for which fuzzy variables have been used.

The experimental results could be reflected qualitatively, but quantitative deviations are still present. The experimental graphs are steeper than the numerical ones. For all three numerically investigated cases, the output fuzzy standard deviations are approximately $10 \%$ of the output fuzzy mean values by what the influence of the stochastic input parameters becomes visible. The influence of the fuzzy input parameters can be seen for example in the support intervals of the output fuzzy mean values, which 


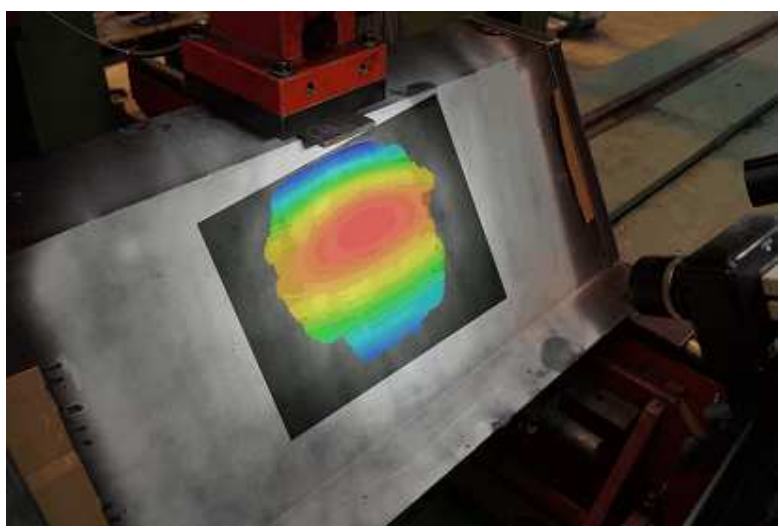

(a) displacement field just before failure

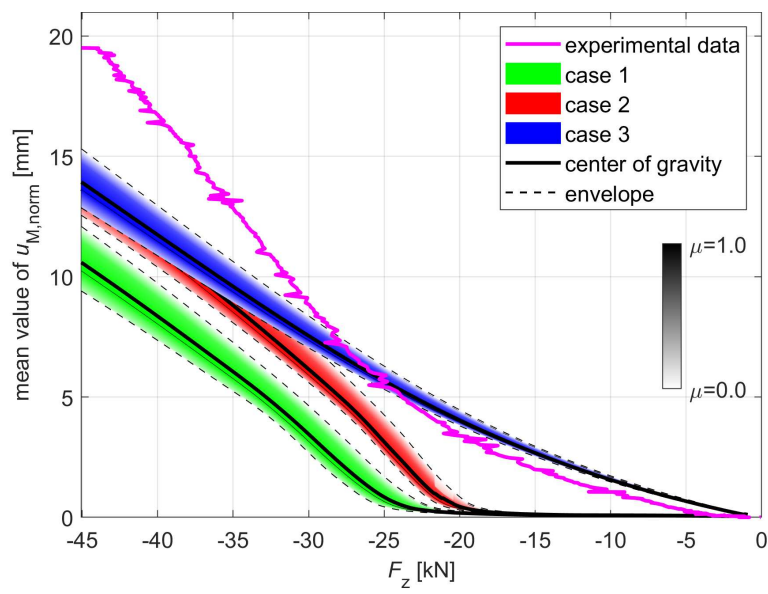

(c) fuzzy mean value of $u_{\mathrm{M} \text {,norm }}$

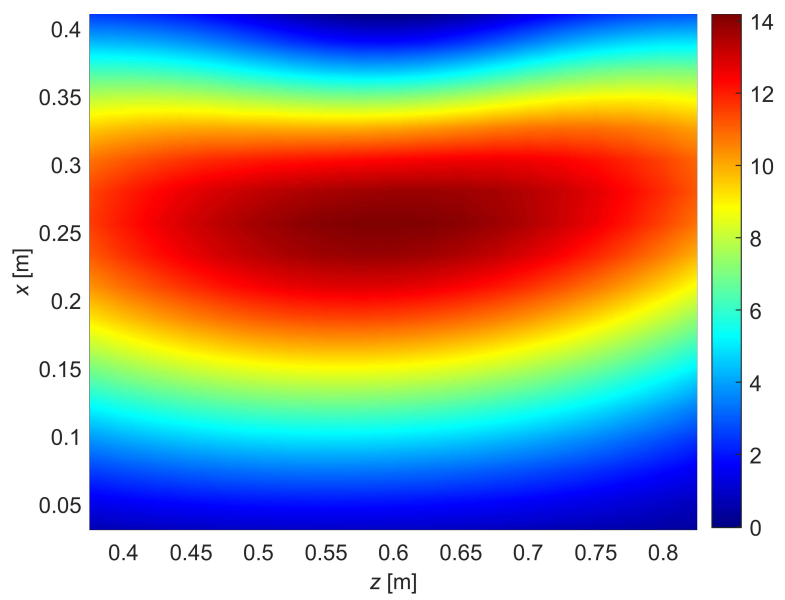

(b) $\operatorname{COG}\left(\right.$ mean value of $\left.u_{\text {norm }}\right)[\mathrm{mm}]$ at $F_{\mathrm{z}}=-45.36 \mathrm{kN}$

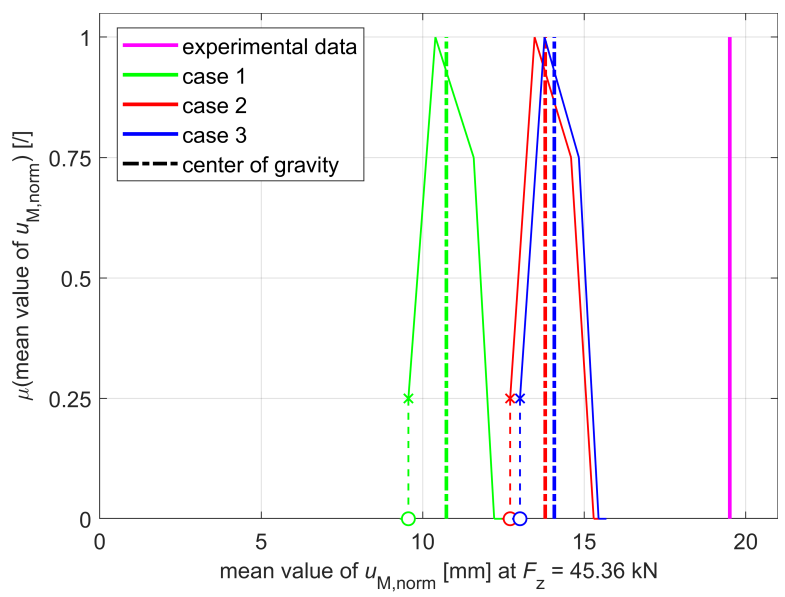

(d) membership function at $F_{\mathrm{z}}=-45.36 \mathrm{kN}$

Figure 10: Fuzzy-stochastic output and experimental result for displacement normal to the web surface

are approximately $\pm 10 \%$ around the associated centers of gravity. The reduction of the fiber volume content from $55 \%$ to $47 \%$ in case 2 decreases the stiffness of the structure and decreases the deviation to the experimentally determined strains and displacements by approximately $37 \%$. In addition, the predeformation according to the symmetrical buckling mode in case 3 decreases the deviation once more and the experimental graph can be better retraced, especially for smaller load values.

\section{ACKNOWLEDGEMENT}

The authors gratefully acknowledge the financial support of the German Research Foundation (DFG) within the Subproject 4 (grant number: 312928137) of the Priority Programme "Polymorphic uncertainty modelling for the numerical design of structures - SPP 1886" and the valuable contribution of IPF Dresden during the design and manufacturing phase. 


\section{REFERENCES}

[1] Brøndsted, P. and Nijssen, R. P. L. Advances in wind turbine blade design and materials. Woodhead Publishing Series in Energy, (2013).

[2] Castro, S.G.P., Zimmermann, R., Arbelo, M.A. et al. Geometric imperfections and lower-bound methods used to calculate knock-down factors for axially compressed composite cylindrical shells Thin-Walled Structures (2014) 74:118-132.

[3] Ditcher, A.K., Rhodes, F.E. and Webber, J.P.H. Non-linear stress-strain behaviour of carbon fibre reinforced plastic laminates The Journal of Strain Analysis for Engineering Design (1981) 16(1):43-51.

[4] Drieschner, M., Eichner, L., Seiffarth, F. and Petryna, Y. PolyUQ: framework for solving problems with polymorphic uncertainties. Preprint-Reihe des Fachgebiets Statik und Dynamik, Technische Universität Berlin, Preprint No. 2021-01, (2021).

[5] Flügge, W. Die Stabilität der Kreiszylinderschale Archive of Applied Mechanics 3.5, 463-506, Springer-Verlag, (1932).

[6] Hanss, M. Applied Fuzzy Arithmetic - An Introduction with Engineering Applications. SpringerVerlag Berlin Heidelberg, (2005).

[7] Khakimova, R., Wilckens, D., Reichardt, J. et al. Buckling of axially compressed CFRP cylinders with and without additional lateral load: Experimental and numerical investigation Thin-Walled Structures (2017) 119:178-189.

[8] Kiureghian, A.D. and Ditlevsen, O. Aleatory or epistemic? Does it matter? Structural Safety (2009) 31(2):105-112.

[9] R\&G Faserverbundstuffe GmbH Materialdatenblätter Epoxidharz L 20 + Härter EPH 161. (2019).

[10] Schürmann, H. Konstruieren mit Faser-Kunststoff-Verbunden. Springer-Verlag Berlin, Vol. II., (2007).

[11] Teijin Carbon Europe GmbH Produktübersicht Kohlenstofffaser Filamentgarn. (2019).

[12] VDI Development of FRP components (fibre-reinforced plastics): Analysis (2014-3). Beuth Verlag $\mathrm{GmbH}$, (2006).

[13] Verwimp, E., Tysmans, T., Mollaert, M. et al. Experimental and numerical buckling analysis of a thin TRC dome Thin-Walled Structures (2015) 94:89-97.

[14] Wolf, C. Messtechnische Erfassung und Modellierung von Imperfektionen und Stabilität dünnwandiger CFK-Bauteile. Master thesis TU Berlin, (2019).

[15] Zhang, S. Experimental identification of statistical correlation between mechanical properties of FRP composite. Materials (2020) 13:674. 